\title{
DIAGNÓSTICO INCIDENTAL DE TUMOR VESICAL POR ECOGRAFÍA GINECOLÓGICA TRANSVAGINAL
}

\author{
Masami Yamamoto C. ${ }^{1}$, Mercedes de Alvarado R. ${ }^{a}$, Manuel Figueroa G. ${ }^{a}$, Juan Pablo \\ Barroso S. ${ }^{2}$, Luis Alberto Caicedo. ${ }^{1}$, Jorge Carrillo T. ${ }^{1}$, Alvaro Insunza F. ${ }^{1}$, Enrique \\ Paiva W. ${ }^{1}$ \\ 1 Unidad de Gestión Clínica de la Mujer y Recién Nacido, ${ }^{2}$ Departamento de Urología, Unidad de Gestión Clínica del \\ Adulto, Hospital Padre Hurtado, Facultad de Medicina Clínica Alemana-Universidad del Desarrollo.
}

a Alumnos, Facultad de Medicina, Universidad del Desarrollo.

\section{RESUMEN}

Se presenta el caso clínico de una mujer de 60 años que acude al Servicio de Ginecología y Obstetricia del Hospital Padre Hurtado, bajo la sospecha de un mioma cervical pequeño. Se realiza una ecografía transvaginal la cual evidencia incidentalmente un tumor vesical sólido, vascularizado, intravesical y dependiente de la mucosa. La paciente no refería espontáneamente hematuria. El estudio urológico definitivo es de un carcinoma papilar de células transicionales. El tratamiento endoscópico logró la resección completa del tumor. El hallazgo ecográfico permitió realizar el diagnóstico en una forma poco habitual para esta enfermedad, aprovechando una instancia definida para otros fines. Se rescata la importancia de observar detenidamente todas las estructuras pélvicas al alcance visual del equipo de ecografía.

PALABRAS CLAVE: Ecografía transvaginal, diagnóstico, tumor vesical, cáncer vesical

\section{SUMMARY}

We present a clinical case of a 60 year-old patient, who concurs to the Obstetrics and Gynecology Department of the Padre Hurtado Hospital under the suspect of a uterine-cervical myoma. A transvaginal ultrasonography was performed which incidentally revealed a bladder tumor which was solid, vascularized, clearly inside of the bladder and attached to the bladder mucosa. The definitive urological study was compatible with a transitional cells papillary carcinoma. The woman did not refer hematuria spontaneously. The finding allowed an unusual diagnosis in an ultrasound aimed for different purposes, which underlines the potential use of this method in other pelvic organs.

KEY WORDS: Transvaginal ultrasonography, diagnosis, bladder tumor, bladder cancer 


\section{INTRODUCCIÓN}

El cáncer de vejiga ocupa el segundo lugar en frecuencia de los cánceres urológicos, la edad promedio de presentación es 65 años y un $25 \%$ se presentan con enfermedad extravesical o metastásica. Son factores de riesgo el tabaquismo, el uso de ciclofosfamida, radioterapia, irritación crónica por infecciones e instrumentación (1).

La edad de presentación habitual es entre 50 y 80 años, la clínica se caracteriza por hematuria macro o microscópica, y en ocasiones la única forma de presentación son los síntomas irritativos urinarios (2). El estudio inicial incluye una citología urinaria y una cistoscopia (3). Con estas herramientas incluso un $25 \%$ de los pacientes presentan un estadio invasor de la muscular, en que la sobrevida no supera el $50 \%$ a 5 años (4). No existe evidencia de calidad que avale un método eficaz de pesquisa (5), y en etapas invasoras la terapia es la cistectomía radical (4). El tratamiento considera el uso de BCG intravesical (6).

En Chile la incidencia de cáncer vesical es de 1,6/100.000 habitantes (7). En el 2003, fallecieron 114 mujeres a causa de esta patología, ocupando el $19^{\circ}$ lugar dentro de las causas oncológicas de muerte en mujeres chilenas con una mortalidad de 1,4/100.000 mujeres, 40\% más que en 1997 (8).

En este reporte se documenta el diagnóstico incidental de un tumor vesical mediante ecografía ginecológica transvaginal, lo que permite fundamentar la importancia que los ecografistas ginecológos no se limiten a observar solamente los genitales internos, y a su vez, demostrar la capacidad de un método diagnóstico muy frecuente para patologías pelvianas en general.

\section{Caso clínico}

Paciente de 60 años, con antecedentes mórbidos de tabaquismo, hipertensión arterial e hipotiroidismo por tiroidectomía y en tratamiento médico. Multípara de 7 partos vaginales, menopáusica hace 22 años, sin terapia de reemplazo hormonal, ooforectomía izquierda por quiste benigno en 1984. Prolapso uterino operado con fijación a ligamentos uterosacros e incontinencia de esfuerzo reparada por TOT en 2003.

Es derivada desde la atención primaria por patología cervical. Ingresa a la Unidad de Patología Cervical del Hospital Padre Hurtado en octubre de 2008 , sin sintomatología ginecológica. A la especuloscopia destacó un cuello de $3 \mathrm{~cm}$ que en labio anterior tenía un nódulo de $1,5 \mathrm{~cm}$ de diámetro, compatible con mioma cervical.
Se realizó ecografía transvaginal cuyos hallazgos fueron: útero en anteversoflexión con dimensiones de $62 \times 30 \times 35 \mathrm{~mm}$ en su diámetro longitudinal, anteroposterior y transversal, respectivamente. Miometrio de aspecto homogéneo. Endometrio de ecogenicidad homogénea, con línea endometrial de $8 \mathrm{~mm}$. Cavidad uterina de aspecto normal. Cérvix con nódulo sólido, ecorrefringente de $28 \times 22$ $\mathrm{mm}$. Ovario derecho de $17 \times 12 \mathrm{~mm}$ de aspecto normal, e izquierdo no se logró observar, sin lesiones anexiales. El fondo de saco de Douglas se apreció libre. En la vejiga se observó un tumor intravesical de $32 \times 25 \mathrm{~mm}$ con bordes irregulares y aspecto irradiado, con hallazgo de vascularización al doppler color (Figuras 1 y 2). Conclusión ecográfica: mioma cervical y tumor vesical en estudio. Se derivó la paciente a urología.

La evaluación urológica destacó la incontinencia urinaria de esfuerzo. El interrogatorio dirigido reveló una hematuria macroscópica intermitente de 2 meses de evolución. La cistoscopia diagnóstica demostró un gran tumor intravesical sésil, de $4 \mathrm{~cm}$ de diámetro aproximado, que nacía sobre el orificio ureteral izquierdo sin comprometerlo, sin sangrado ni otros tumores.

Se planificó una resección transuretral (RTU), su condición prequirúrgica era asintomática, sin hematuria macro ni microscópica y el resto de los exámenes preoperatorios normales. La RTU obtuvo en forma completa el tumor, tomando muestras del fondo y borde que se enviaron a biopsia. La paciente evolucionó favorablemente, destacando sólo algunos coágulos pequeños en sonda Foley, que

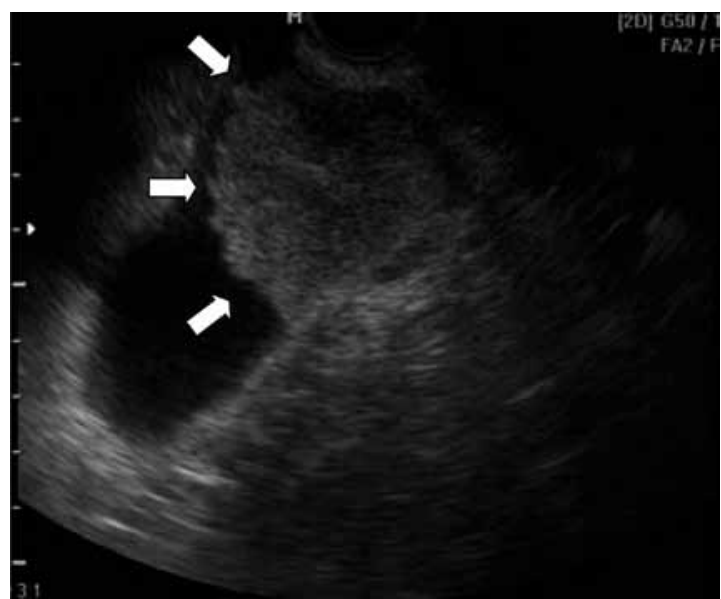

Figura 1. Imagen ecográfica transvaginal en dos dimensiones de vejiga, que evidencia ocupación del lumen vesical por tumor de ecogenicidad heterogénea y bordes irregulares (fechas). 


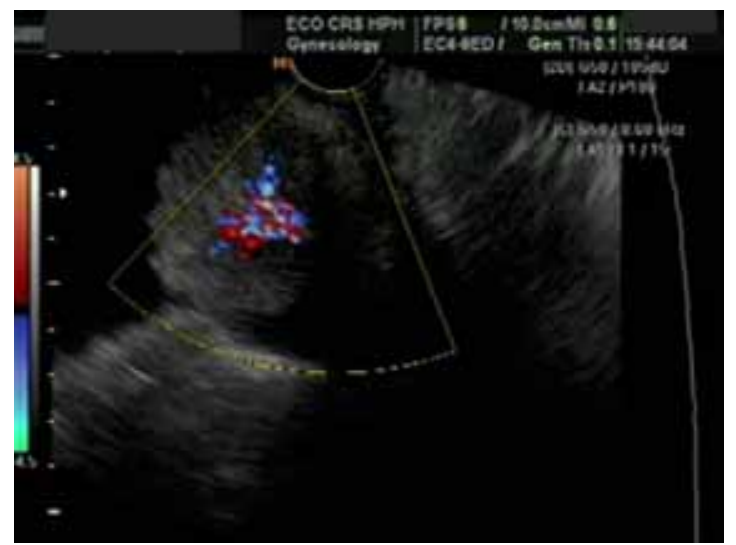

Figura 2. Imagen ecográfica transvaginal en dos dimensiones y doppler color de vejiga, que muestra la vascularización desde el centro. Esta imagen permitió confirmar que era tejido y no coágulo.

requirieron irrigación vesical, cesando al tercer día postoperatorio. En el control urológico posterior la paciente estaba asintomática y sin hematuria.

La biopsia informó un carcinoma urotelial (transicional) de mucosa vesical, de tipo papilar de bajo grado e invasión hasta lámina propia. La muestra de los bordes y fondo del tumor no presentaron invasión.

\section{DISCUSIÓN}

La ultrasonografía transvaginal presenta una utilidad sustantiva para evaluar los órganos pelvianos femeninos. Sin embargo, su máximo desempeño se limita a los diagnósticos gineobstétricos debido a que es el mayor interés de los profesionales que la realizan.

Si bien la mayoría de los trastornos hemorragíparos en la mujer se deben a causa ginecológica, las patologías no ginecológicas deben ser descartadas

Dentro de los hallazgos urológicos en ecografía transvaginal ginecológica se ha descrito la identificación de cáncer vesical, cálculos, coágulos, cuerpos extraños, endometriosis y quistes hemorrágicos. Para identificar lesiones intravesicales, se ha recomendado realizar el examen con vejiga llena o al menos con $50 \mathrm{cc}$ de orina, lo cual proporcionaría una mejor ventana ecográfica e interfase sólidolíquida.

Existen 2 reportes de diagnóstico ecográfico transvaginal de cáncer vesical publicados en 2005 y 2008 , donde se analiza la utilidad, sobre todo en mujeres postmenopáusicas, de esta herramienta diagnóstica, menos invasiva y más económica que la actual, que podría aportar información respecto al grado de invasión parietal y por ende ser útil en la planificación quirúrgica $(9,10)$.

Se ha documentado la utilidad de la ultrasonografía abdominal por radiólogos en la pesquisa de tumores vesicales, siendo mejor que la urografía endovenosa. Un estudio determinó una sensibilidad y especificidad de $99 \%$ y $63 \%$, respectivamente, de la ecografía abdominal, para diagnóstico de tumor vesical en 1007 pacientes con hematuria (11). Las imágenes sugerentes de malignidad son de lesiones sólidas, de bordes irregulares y alta vascularización al Doppler (9).

En nuestro país no existen normas establecidas que detallen indicaciones y utilidad real de esta técnica en el diagnóstico de patologías urológicas.

Este caso clínico muestra la factibilidad del diagnóstico de patologías vesicales por la ecografía transvaginal, pero que pueden pasar inadvertidos por no centrar el interés de la observación fuera de los órganos genitales internos. El reporte rescata la importancia de que el ecografista observe detenidamente no sólo el útero y los anexos, sino todo lo que esté al alcance de la visión sonográfica. El examen por ecografía transvaginal se ha extendido importantemente en el país, por lo que esta instancia de evaluación podría contribuir al manejo de enfermedades no ginecológicas.

\section{BIBLIOGRAFÍA}

1. Damjanov I. El tracto urinario inferior y sistema reproductivo masculino. En: Rubin E, Gorstein F, Rubin R, Schwarting R, Strayer D. Patología Estructural. Fundamentos Clínico Patológicos en Medicina. 4a ed. Madrid, McGraw-Hill \& Interamericana, 2006; 817-21.

2. Epstein Jl. Tracto urinario inferior y sistema genital masculino. En: Kumar V, Abbas A, Fausto N. Robbins y Cotran. Patología Estructural y Funcional. $7^{\mathrm{a}}$ ed. Madrid: Elsevier, 2005; 1030 -6.

3. NCCN. Clinical Practice Guidelines in Oncology. Bladder cancer including upper truct tumors and urothelial carcinoma of the prostate; 2009.

4. Torrealba J, Muñoz P, Dell'Oro A, Pinochet R. Sobrevida del cáncer vesical infiltrante. Rev Chil Urol 2008; 73(4):292-7.

5. U.S. Preventive Services Task Force. Screening for Bladder Cancer: Brief Evidence Update. June 2004. Agency for Healthcare Research and Quality, RockviIle, MD.

6. Shelley M, Court JB, Kynaston H, Wilt T, Fish R, Mason M. Intravesical Bacillus Calmette-Guérin in $\mathrm{Ta}$ and T1 bladder cancer. Cochrane Database of Systematic Reviews 2000, Issue 4. Art. No.: CD001986. DOI:10.1002/14651858.CD001986

7. Medina E, Kaemnffer A. Mortalidad por cáncer en 
Chile: consideraciones epidemiológicas. Rev Méd Chile 2001;129(10):1195-202.

8. Donoso E, Cuello M. Mortalidad por cáncer en la mujer chilena. Análisis comparativo entre los años 1997 y 2003. Rev Chil Obstet Ginecol 2006;71(1):10-6.

9. Betsas G, Van den Bosch T, Deprest J, Bourne T, Timmerman $\mathrm{D}$. The use of transvaginal ultrasonography to diagnose bladder carcinoma in women presenting with postmenopausal bleeding. Ultrasound Obstet Gy- necol 2008;32:959-60.

10. Huang WC, Yang SH, Yang JM. Three-dimensional ultrasonographic findings in bladder cancer. Ultrasound Obstet Gynecol 2005;25:90-4.

11. Datta SN, Allen FM, Evans R, Vaughton KC, Lucas MG. Urinary tract ultrasonography in the evaluation of haematuria - a report of over 1,000 cases. Ann R Coll Surg Engl 2002;84:203-5. 